PROFESIONALES Y HERRAMIENTAS PARA EL DESARROLLO LOCAL Y SUS SINERGIAS TERRITORIALES. EVALUACIÓN Y PROPUESTAS DE FUTURO IX Coloquio Nacional de Desarrollo Local del GTDL-AGE 

ANTONIO MARTÍNEZ PUCHE, XAVIER AMAT MONTESINOS, ISABEL SANCHO CARBONELL y DANIEL SANCHIZ CASTAÑO (EDS.)

\section{PROFESIONALES Y HERRAMIENTAS PARA EL DESARROLLO LOCAL Y SUS SINERGIAS TERRITORIALES. EVALUACIÓN Y PROPUESTAS DE FUTURO}

IX Coloquio Nacional de Desarrollo Local del GTDL-AGE

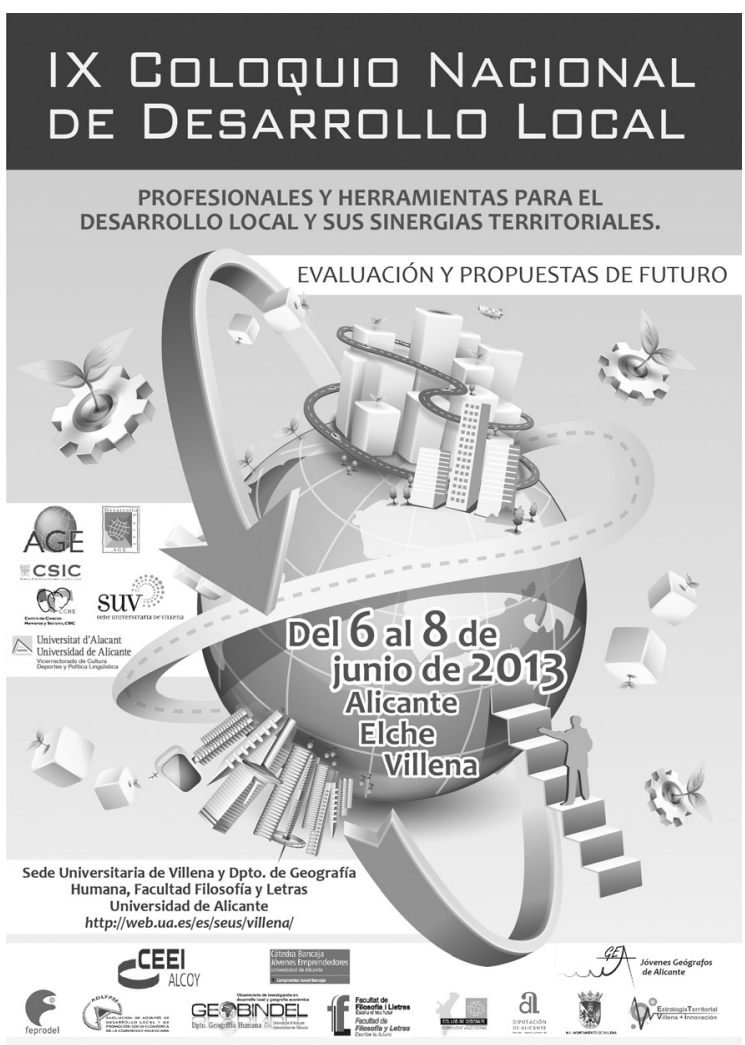


Este libro ha sido examinado y valorado por evaluadores ajenos a la Universidad de Alicante, con el fin de garantizar la calidad científica del mismo.

Publicacions de la Universitat d'Alacant

03690 Sant Vicent del Raspeig

Publicaciones@ua.es

http://publicaciones.ua.es

Telèfon: 965903480

(C) Antonio Martínez Puche, Xavier Amat Montesinos,

Isabel Sancho Carbonell y Daniel Sanchiz Castaño (eds.), 2016

(C) d'aquesta edició: Universitat d'Alacant

ISBN: 978-84-16724-00-0

Dipòsit legal: A 92-2016

Disseny de coberta: candela ink

Composició: Página Maestra (Miguel Ángel Sánchez Hernández)

Impressió i enquadernació: Guada Impresores

\section{unte \\ Unión de Editoriales
Universitarias Españolas \\ WWW.une.es
WWA}

Esta editorial es miembro de la UNE, cosa que garantiza la difusión y comercialización nacional y internacional de sus publicaciones.

Reservados todos los derechos. Cualquier forma de reproducción, distribución, comunicación pública o transformación de esta obra sólo puede ser realizada con la autorización de sus titulares, salvo excepción prevista por la ley. Diríjase a CEDRO (Centro Español de Derechos Repográficos, www.cedro.org) si necesita fotocopias o escanear algún fragmento de esta obra. 


\title{
LA OBRA SOCIAL DE CAIXA ONTINYENT COMO DINAMIZADORA DEL DESARROLLO LOCAL EN LA COMARCA DE LA VALL D'ALBAIDA
}

\author{
Eva $M^{a}$ Silvestre Francés \\ Alumna Máster Oficial Desarrollo Local e Innovación Territorial, UA \\ emsf1@alu.ua.es
}

\section{RESUMEN}

La comarca de la Vall d'Albaida posee uno de los agentes socioeconómicos más importante y de referencia en la actualidad dentro de sistema financiero nacional, es Caixa Ontinyent, la cual aporta un valor añadido a la comarca y diferenciación del resto de territorios. Caixa Ontinyent cuenta con una antigüedad de más de 128 años llevando a cabo su actividad económico-financiera y su labor social, dicha entidad se creó por y para la sociedad siguiendo esta línea hasta día de hoy. La Obra Social de Caixa Ontinyent es el instrumento fundamental, a través de la cual ésta entidad devuelve a la sociedad los beneficios generados de su actividad financiera. La Obra Social se convierte en uno de los componentes esenciales para los municipios de la comarca.

Palabras clave: Obra social, Caja de Ahorros, territorio, desarrollo, servicios.

\section{SOCIAL WORK CAIXA ONTINYENT AS ENGINE OF LOCAL DEVELOPMENT IN A REGION OF THE VALL DE ALBAIDA}

\section{ABSTRACT}

The Albaida Valley area has one of the most important socioeconomic agents and nowadays is a reference within the national financial system: Caixa Ontinyent, which contributes an added value to the region, making it different from the rest of the regions. Caixa Ontinyent has been carrying out its economic financial activity and social work for more than 128 years. The company was created by and for the society, continuing this line of action until the present day. The 
Welfare Project of Caixa Ontinyent is the main instrument through which this company gives back the profits generated through its financial activity to society. The social work is one of the essential components for the towns in the region.

Key words: Welfare Project, savings bank, territory, local development, services.

\section{Caracterización territorial comarca Vall d’Albaida}

La comarca de la Vall d'Albaida se encuentra situada en la Comunidad Valenciana, en el interior de la provincia de Valencia, lindando con el norte de la provincia de Alicante. La Vall d'Albaida está rodeada por las comarcas de La Costera, La Safor, El Comtat, L'Alcoiá i el Alt Vinalopó, todas ellas conforman lo que conocemos como comarques centrals valencianes (a excepción de l'Alt Vinalopó). La Vall d'Albaida posee una extensión territorial de 722,2 km² con un total de población según el Padrón Municipal a 1 de Enero de 2012 de 92.106 habitantes, la comarca se componte de 24 municipios, como cabecera comarcal Ontinyent municipio el cual concentra el mayor número de población con 37.140 habitantes, supone un porcentaje del $40 \%$ sobre el total comarcal. Por detrás de Ontinyent existen 5 municipios que poseen una población entre 9.000 y 4.000 habitantes (Olleria (9\%), Benigànim (7\%), Albaida (7\%), Aielo de Malferit (5\%) y Bocairent $(5 \%), 9$ municipios con una población entre 3.999 y 1.000 habitantes (Pobla del Duc, Lutxent, Quatretonda, Agullent, Castelló de Rugat, Montaverner, Alfarrasí, Atzeneta D'Albaida y Fontanars dels Alforins), y por último 19 municipios con menos de 1.000 habitantes, estos municipios con un bajo porcentaje de representatividad sobre el total de población comarcal (Bèlgida, Benicolet, Montixelvo, Palomar, Otos, Salem, Guadasséquies, Ráfol de Salem, Benissoda Bellús, Terrateig, Beniatjar, Benisuera, Rugat, Aielo de Rugat, Bufali, Pinet, Carrícola y Sempere).

El motor económico que ha caracterizado a la comarca ha sido alrededor del sector industrial, con un predominio del sector textil para el hogar, en la Estrategia Territorial de la Comunidad Valenciana 2010-2030 se expone que "La Vall d'Albaida es un territorio de tradición industrial, en torno al cluster del sector textil, cuyo desarrollo en el siglo pasado le ha permitido fijar su población en el territorio y atraer inmigración laboral, tanto interna como externa, haciendo de Ontinyent uno de los nodos claves para el mantenimiento de la estructura territorial de la Comunitat".

La cultura (tangible e intangible) de la comarca es un aspecto fundamental a la hora de apreciar la unión entre los diferentes municipios de la comarca. La Vall d'Albaida mantiene sus tradiciones vinculadas directamente a su historia, aportando una singular identidad al territorio y a su población. 


\section{Historia y nacimiento de Caixa Ontinyent}

Hablar de Caixa Ontinyent y de su Obra Social, no tiene sentido sin mencionar su origen histórico y su trayectoria, entidad que a día de hoy cuenta con una antigüedad de 128 años. Esta Caja nace en 1884 en el municipio de Ontinyent como proyecto anexo y bajo el amparo de la Sociedad de Socorros Mutuos La Previsora (1881).

En el s. XIX no existía cobertura ni prestaciones sociales a los trabajadores, fueron las Sociedades de Socorros Mutuos los encargadas de ofrecer a sus socios que se adherían a estas sociedades las coberturas y prestaciones sociales cuando el trabajador asociado se encontrara en situación desfavorable, tanto por baja laboral y en caso de defunción una prestación monetaria a la familia del asociado, en este sentido trabajaba La Previsora.

Tres años después del nacimiento de La Previsora, nace La Caja de Ahorros y Monte de Piedad de Onteniente, "La Previsora fundó fuera de su marco la Caja aunque se convertiría en su principal imponente inicial y por tanto, verdadero motor de la entidad naciente" (BERNABEU, LÓPEZ, TITOS, PLAZA, TORTOSA, 1984). La reciente inaugurada Caja de Ahorros y Monte de Piedad de Ontinyent se creó para trabajar ofrecer sus servicios a la clase obrera y a toda la población del municipio de Ontinyent, es decir una Caja al servicio de la población. La Caja de Ahorros y Monte de Piedad de Onteniente tenía las funciones de una Caja de Ahorro: fomentando la rentabilidad de los ahorros de sus clientes; y el del Monte de Piedad: prestamos de garantía prendaría, el cual permitía a que las clases más pobres tuvieran acceso al crédito a través del Monte de Piedad, (SANCHIS, ORTIZ, SANZ, SANCHIS, BENEITO, 2008).

La Previsora y La Caja de Ahorros y Monte de Piedad de Onteniente seguirían su transcurso en paralelo, pero La Previsora era la que se quedaba los grandes beneficios que producía la Caja de Ahorros. Ambas entidades tuvieron que ir adaptándose a las graves dificultades de guerra y de postguerra que se dan el s.XX.

Fue en 1948, año en que se concluye La Previsora, debido a la perdida de socios por la aparición de dos seguros públicos: el Seguro de Enfermedad en 1942 "destinado a cubrir la asistencia sanitaria completa y la indemnización económica (la mitad del salario) en los percances de enfermedad y maternidad; el seguro proporcionaba cobertura a todos los trabajadores manuales y a los trabajadores no manuales" (COMÍN, 2012) y el Seguro Obligatorio de Vejez e Invalidez en 1947.

A partir de este punto, la Caja de Ahorros y Monte de Piedad sigue su trayectoria fortaleciendo su trabajo y adaptando sus funciones dentro del marco legal que le corresponde para seguir su trayectoria en el mundo de las Cajas de Ahorros. 


\section{NUESTRAS CAJAS DE AHORROS}

Desde la década de los 70 hasta llegar a la actualidad, la figura de nuestras Cajas de Ahorro españolas han estado sometidas a profundos cambios, es decir, los procesos de fusión, transformación e incluso de desaparición, son hecho de gran relevancia en el panorama económico y financiero de nuestro país. Estos cambios producidos en las Cajas han desembocado en una reducción del número de Cajas de Ahorro españolas.

En 1989 aparece en el contexto de las Cajas de Ahorro "existencia de un marco liberalizado para la expansión geográfica" (CALS, 2005), momento que empieza la expansión R. Decreto 1582/1988 de 29 de diciembre, comienza a darse competencia entre ellas, también empiezan a actuar como bancos dando lugar al acelerado proceso de disminución de las Cajas debido a su expansiónfusión, la escala de actuación de las Cajas empieza variar de local a regional, de regional a nacional.

En la Comunitat Valenciana, Murcia y Albacete (Federación de Levante) inicialmente existía un total de 16 Cajas de Ahorros (LÓPEZ, 1973), que te-

\section{Cuadro $n^{\circ} 1$. Evolución en las Cajas de Ahorros de la Comunitat Valenciana y} Murcia hasta la actualidad

\begin{tabular}{|c|c|c|}
\hline $\begin{array}{c}\text { - Monte de Piedad y Caja de Ahorros de Alcoy } \\
\text { - Caja de Ahorros y socorros y Monte de Piedad Nuestra } \\
\text { Señora de los Dolores (de Crevillente) } \\
\text { - Caja de Ahorros de Novelda } \\
\text { - Caja de Ahorros y Socorros y Monte de Piedad Nuestra } \\
\text { Señora de Monserrate (Orihuela) } \\
\text { - Caja de Ahorros del Sureste de España (Alicante) } \\
\text { - Caja de Ahorros de Alhama de Murcia } \\
\text { - Caja de Ahorros de Murcia } \\
\text { - Caja de Ahorros y Monte de Piedad de Torrent } \\
\text { - Caja de Ahorros Provincial de Alicante y Valencia }\end{array}$ & $\begin{array}{c}\text { Caja de } \\
\text { Alicante y } \\
\text { Murcia } \\
\text { Caja de } \\
\text { Ahorros del } \\
\text { Mediterráneo } \\
\text { CAM }\end{array}$ & $\begin{array}{l}\text { Banco Sabadell y } \\
\text { CAM: } \\
\text { SabadellCAM }\end{array}$ \\
\hline $\begin{array}{l}\text { - Caja de Ahorros y Monte de Piedad de Valencia } \\
\text { - Caja de Ahorros y Monte de Piedad de Segorbe } \\
\text { - Caja de Ahorros y Monte de Piedad de Castellón } \\
\text { - Caja de Caja de Ahorros y Socorros de Sagunto } \\
\text { - Caja de Ahorros y Préstamos de Carlet }\end{array}$ & $\begin{array}{l}\text { Caja de } \\
\text { Valencia, } \\
\text { Castellón y } \\
\text { Alicante: } \\
\text { (Bancaja) }\end{array}$ & $\begin{array}{c}\text {-Bancaja } \\
\text {-Caja Madrid } \\
\text {-Caja de Canarias } \\
\text { - Caixa Laietana } \\
\text {-Caja de Ávila } \\
\text {-Caja Segovia } \\
\text {-Caja Rioja } \\
\text { Bankia }\end{array}$ \\
\hline Caja de Ahorros y Monte de Piedad de Onteniente $\rightarrow$ & Caixa Ontinyent & \\
\hline
\end{tabular}

Fuente: elaboración propia a partir de J. Cals (2005) y J. López (1973).

$(\rightarrow$ : Cambio de nombre) 
nían un ámbito de actuación localizado en su propio territorio, pero que han ido sumándose a los cambios producidos en el contexto financiero de las cajas, hasta contar en la actualidad con 3 entidades, SabadellCAM y Bankia con un gran ámbito de actuación, mientras que Caixa Ontinyent queda localizada en su ámbito de actuación en les comarques centrals valencianes. El cuadro $\mathrm{n}^{\circ} 1$ muestra la evolución de nuestras cajas hasta la actualidad.

Según datos de la CECA en su Informe publicado a 8 febrero de este mismo año (2013) "de un total de 45 Cajas de Ahorros (a comienzos de 2010), 43 han participado o se encuentran participando en algún proceso de consolidación, lo que en volumen de activos totales medios representa el 99,9\% del Sector", entre las dos Cajas de Ahorros que no han estado sometidas a ningún proceso de cambio a nivel nacional son Caixa Ontinyent y Caixa Pollença.

A partir de lo expuesto en el cuadro $n^{\circ} 1$, observamos que la tendencia ha sido la de concentración y fusión en las Cajas de esta Comunidad, llegando a una pérdida de la esencia en el origen de las Cajas, las cuales poseían una gestión vinculada a su territorio, más conservadora y transmisora de seguridad a los clientes para el fomento del ahorro.

\subsection{Caixa Ontinyent en clave territorial}

Las Cajas de Ahorro han estado fuertemente ligadas a su territorio en su origen, este ha sido uno de los factores más importantes en el modelo de nuestras cajas, ya que las Cajas nacían en tu territorio determinado, se desarrollaban y actuaban en él proporcionando desarrollo económico y social en su propio territorio, J. Cals (2005) apunta que las Cajas inicialmente "facilitaban el acceso a los servicios financieros para los colectivos desfavorecidos, apoyo al desarrollo económico de la zona de actuación y reversión a la sociedad local parte de los beneficios obtenidos". Esta vinculación territorial de las Cajas ha variado, como antes he mencionado la escala de actuación de las Cajas ha aumentado derivando hacia una pérdida del sentido de pertenencia territorial. La concentración de la Cajas en pocas entidades pero de gran tamaño supone que muchas oficinas cierren y zonas rurales, pueblos pequeños, personas, etc, van a perder esta proximidad con su Caja de Ahorros de referencia.

Caixa Ontinyent es un referente de Caja de Ahorros vinculada a su territorio, principalmente en la Vall d'Albaida. El hecho ser una Caja de proximidad ha supuesto ser un valor añadido en el modelo de esta Caja y uno de los pilares fundamentales en su funcionamiento.

La vinculación territorial que posee Caixa Ontinyent le aporta un conocimiento profundo de su entorno y de los agentes socioeconómicos que componen el territorio y operan en él (conocimiento de las personas), ambas son circunstancias indispensables: para dotar de financiación a las actividades que se desarrollen en el territorio; disminuir la exclusión financiera de los munici- 
pios de la comarca, y para retornar los beneficios obtenidos a través de Obra Social procedentes de la actividad financiera.

Al poseer un territorio de actuación concentrado da lugar a que exista conocimiento personal entre la entidad y sus cliente, Caixa Ontinyent conoce a todos sus clientes personalmente. Las tomas de decisiones se dan en el propio territorio, la cual cosa hace que se reconsideren muchos aspectos debido al conocimiento personal con el propio cliente, mientras que por el contrario otras entidades al estar expandidas por todo el país la toma de decisiones se da a cientos de kilómetros de dónde habita el cliente y no suponen un contacto directo éste.

\subsubsection{La Red de Oficinas de Caixa Ontinyent}

La red de oficinas de Caixa Ontinyent va a ser un elemento clave a la hora de realizar su Obra Social. Caixa Ontinyent posee una red de sucursales compuesta por un total de 47 oficinas, la expansión en la red de oficinas ha sido muy lenta si consideramos la variable tiempo (desde su origen) y el número de oficinas hasta la actualidad. La comarca de la Vall d'Albaida actualmente cuenta con 24 oficinas, incluyendo a la Central de la entidad como a la División de Empresas de Caixa Ontinyent. El municipio de Ontinyent posee mayor número de oficinas, además de ser la localidad de nacimiento de esta Caja es donde más población se concentra en la comarca. En el siguiente mapa se refleja la distribución de las oficinas de Caixa Ontinyent en los diferentes municipios de la Vall D’Albaida.
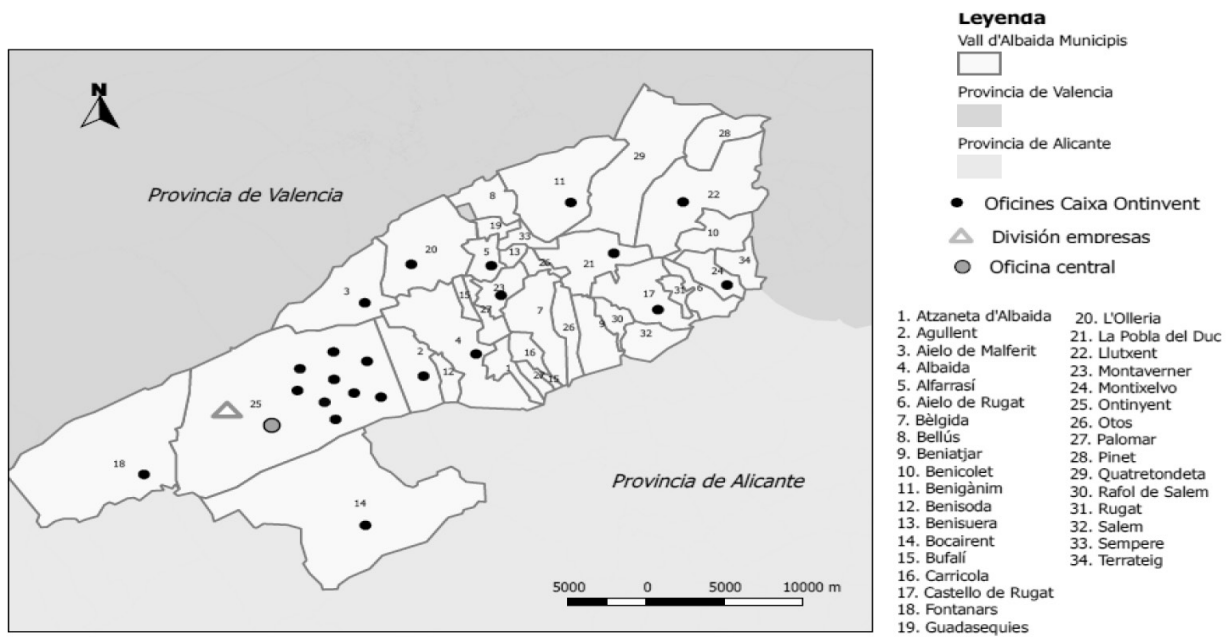

Mapa $n^{o}$ 1. Localización de las Oficinas de Caixa Ontinyent en los municipios de la Comarca de la Vall d'Albaida. Año 2012.

Fuente: Caixa Ontinyent. Elaboración propia. 


\section{LA OBRA SOCIAL DE LAS CAJAS DE AHORRO}

La función social de las Cajas de Ahorros es y ha sido desde siempre uno de los pilares que conforma a nuestras Cajas, éstas no se conciben sin su Obra Social, "la Obra social de las Cajas españolas es consustancial a la misión benéfica y social que inspira su nacimiento y finalidades, las cuales, a su vez, entroncan con la iniciativa benéfica y social de los montes de piedad" (CALS, 2005).

La Obra Social es uno de los componentes más importantes en la actuación de las Cajas de Ahorro, y en algunos casos les ha llevado a que estas acciones sociales sean el elemente clave de anclaje a sus territorios, como es el caso de Caixa Ontinyent. En el Informe publicado por la CECA (Confederación Española de Cajas de Ahorro) en julio del 2012 presenta que la Obra social "personifica, a través de las muy diversas iniciativas desarrolladas, el compromiso social que orienta la actividad de las Cajas de Ahorros".

Las inversiones en Obra Social por parte de las Cajas ha ido cambiando y adaptándose a las necesidades de cada periodo, es decir, empiezan cubriendo una serie de necesidades básicas a la sociedad que no eran atendidas por el Estado y a medida que se desarrolló e implantó el Estado de Bienestar la función social de las Cajas ha ido atendiendo otras necesidades. En la obra del autor Joan Cals (2005) a través de sus estudios se observa que existe una diferenciación importante en cuando a la dotación a obra social y distribución por áreas: en 1978 el 40\% de beneficios eran destinados al área asistencial y el $60 \%$ restante es repartido entre el área docente, cultural, sanitaria e investigación; en 2004 la dotación destinada y distribución por áreas de inversión social se distribuyen de manera diferente: el área de cultura fue la que más inversión recibió con un 50\%, seguido por el asistencial, docente, sanitaria y finalmente Medioambiental.

Esta diferenciación por áreas de actuación es la clasificación que últimamente realiza la CECA como se observa en la Memoria Obra Social 2011, publicada el 25 de julio 2012, distribuye el ámbito de actuación de las Cajas de Ahorros en 4 áreas dirigidas al desarrollo socioeconómico y social del territorio en el que actúan: 
Cuadro $n^{\circ}$ 2. Distribución de áreas de actuación por la Obra Social de las Cajas

\begin{tabular}{|c|}
\hline Cultura y Tiempo Libre: \\
\hline -Cultura \\
\hline -Tiempo libre \\
\hline Asistencia Social y Sanitaria: \\
\hline -Asistencia Social \\
\hline -Sanitaria \\
\hline Educación e Investigación: \\
\hline -Educación \\
\hline -Investigación y Desarrollo \\
\hline Patrimonio Histórico Artístico y Natural: \\
\hline -Patrimonio Histórico Artístico \\
\hline -Patrimonio Natural. \\
\hline
\end{tabular}

Fuente: CECA. Elaboración propia.

\subsection{La Obra Social de Caixa Ontinyent}

La Obra Social de Caixa Ontinyent es una realidad en los municipios de la comarca de la Vall d'Albaida que poseen oficinas de la entidad, contribuyendo al desarrollo local de este territorio. Los autores Calvo, R. y Lerma, I., (2009) realizan una clasificación de los actores sociales locales en base a la relación con el proceso de desarrollo local del municipio o territorio determinado, diferencian los actores que actúan de forma directa en relación a las políticas activas de empleo, y a los indirectos por la importancia de las acciones que realizan en el territorio, en éste último clasifican a la Obra Social.

La filosofía de Caixa Ontinyent en el desempeño de su Obra Social es el reinvertir los beneficios obtenidos de su actividad en la sociedad, es decir, cada pueblo ha de tener en obra social el equivalente a los beneficios que aporta cada oficina. La distribución de oficinas de Caixa Ontinyent en los diversos municipios la comarca (visto en el apartado 3.I.I.) es fundamental para la dotación en obra social, los benéficos de cada oficina se invertirán en Obra Social en ese mismo municipio. Anualmente los beneficios de cada oficina se van acumulando hasta alcanzar la cantidad deseada para poder desarrollar su inversión social.

Caixa Ontinyent realiza dos tipos de Obra social:

- Obra propia: cuando la obra es financiada enteramente por la propia Caja y la inversión, gestión y administración es exclusivamente llevada por Caixa Ontinyent. 
- Obra en Colaboración: cuando existe colaboración con otras instituciones, en este caso Caixa Ontinyent dirigirá su obra en colaboración aportando inversión, bienes o servicios para realizar la actuación conjuntamente con su colaborador.

La realización de la Obra Social de Caixa Ontinyent crea sinergias con diferentes colectivos de la comarca, esta entidad ha mantenido relación directa a través de su Obra social con más de 300 colectivos.

\subsection{Actuaciones de la Obra Social de Caixa Ontinyent}

Caixa Ontinyent ha realizado diversas y amplias actuaciones en Obra Social en la comarca, pero se considera de forma muy especial la Obra Social por excelencia de Caixa Ontinyent: cuando La Previsora (fundadora de Caixa Ontinyent) finiquitó su actividad en el año 1948 "La Sociedad de Socorros Mutuos pasó a ser la obra social más importante de Caixa Ontinyent que asumió los compromisos pensionistas de La Previsora a cambio del edificio que albergaba a las dos entidades" (SANCHIS, ORTIZ, SANZ, SANCHIS, BENEITO, 2008). En la actualidad Caixa Ontinyent continua el compromiso con su fundadora aunque el número de socios con vida de La Previsora cuantitativamente sea muy reducido, pero cualitativamente los significados que ello aporta son innumerables.
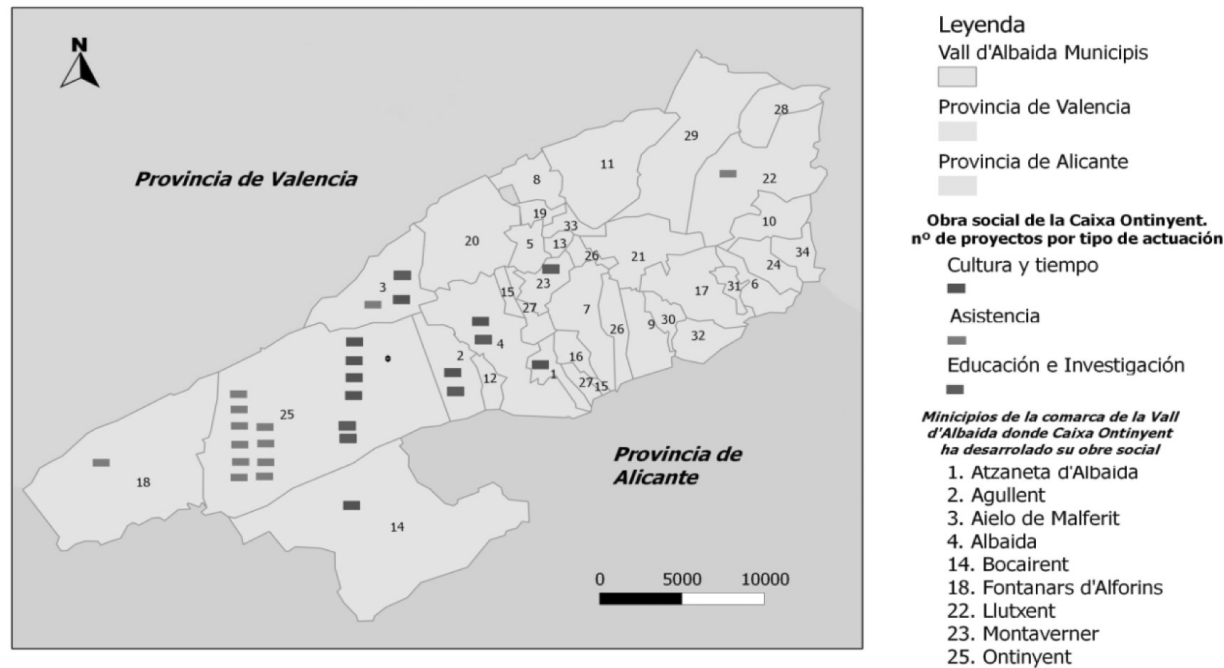

Mapa $n^{\circ}$ 2. La Obra Social de Caixa Ontinyent en los municipios de la comarca de la Vall d'Albaida. Número de proyectos desarrollados según actuación. Año 2012. 
Hasta 1980 la Obra Social de Caixa Ontinyent se concentraba en el municipio de Ontinyent, y es a partir de ésta década cuando empieza a dotar de infraestructura y servicios a los diferentes municipios, se diversifica la dotación por áreas de actuación potenciando más la cultura, sin dejar a un lado la asistencia a los sectores más desfavorecidos. En el mapa $n^{\circ} 2$ se observa la distribución espacial desde el años 1982 al 2012 de las Obras Sociales llevadas a cabo por Caixa Ontinyent, tanto en obra propia como en obra en colaboración con otros agentes.

Como se observa en el mapa $\mathrm{n}^{\circ} 2$ de distribución de Obra social son los municipios que más población concentran los que poseen inversión de Obra social, en el caso del municipio de Ontinyent ( $\left.n^{\circ} 25\right)$ es el que más dotaciones sociales posee, 10 proyectos dentro del área Asistencial, 2 del área de Educación, y 4 de Cultura y Tiempo Libre, a continuación le sigue Aielo de Malferit con 3 actuaciones. Cabe hacer una mención especial al Centro Cultural de Caixa Ontinyent inaugurado en 2010, desde la fecha albergando infinidad de actividades (exposiciones, conciertos, conferencias, ciclos de cine, etc) realizadas por diversos colectivos comarcales, el Centro Cultural de Caixa Ontinyent obtuvo un reconocimiento a nivel nacional en la Revista Actualidad Económica la cual distingue la dinamización de este centro como una de las mejores Obras Sociales del año, recibiendo el tercer premio a la mejor Obra Social de España en el área de actuación de Cultura y Tiempo Libre. Contar con un reconocimiento de tal magnitud refleja la gran labor social y el compromiso asumidos por Caixa Ontinyent con la sociedad.

\section{CONCLUSIÓN}

Caixa Ontinyent se ha convertido en un referente por su correcto funcionamiento en el contexto socioeconómico y financiero de nuestro país. Este modelo de Caja que a través de las buenas prácticas financieras e inversiones llevadas a cabo en toda su trayectoria son el efecto demostrativo, en el cual ha promovido y dinamizado el desarrollo económico y social de la comarca de la Vall d'Albaida. Su gran labor aporta desarrollo territorial y cohesión social en la comarca, debido a que Caixa Ontinyent no ha perdido la esencia que inicialmente dictaminaba el funcionamiento de las Cajas de Ahorro, siempre aplicando la ética y el sentido común.

Caixa Ontinyent con la reinversión de gran parte de sus beneficios a través de su Obra Social mejora la calidad de vida de la población, dota de servicios y su vez proporciona puestos de trabajo vinculados a su actividad directa o indirectamente.

La aportación en infraestructura por parte de la Obra Social de Caixa Ontinyent cuenta con 27 centros los cuales son el reflejo de edificios vivos, que se encuentran en pleno funcionamiento y al servicio de la sociedad, desde su apertura hasta la actualidad. 
El contar con Caixa Ontinyent en la comarca de la Vall d'Albaida hace que aumente el capital social y productivo, y el bienestar de la población, convirtiéndose en un elemento esencial para el desarrollo local.

\section{BIBLIOGRAFÍA}

BERNABEU, A., LÓPEZ, J., TITOS, M., PlAZA, J., TORTOSA, F., (Coord. CECA) (1984): Una institución centenaria. La Caja de Ahorros y Monte de Piedad de Onteniente (1884-1984), Madrid, Caja de Ahorros y Monte de Piedad de Onteniente, p. 438.

CAIXA ONTINYENT: http://caixa.caixaontinyent.es/ , [consulta: 5/06/2012]

CALS, J., (2005): El éxito de las Cajas de Ahorro. Historia reciente, estrategia competitiva y gobierno, Barcelona, Ariel, p 231.

CALVO, R., LERMA, I. (2009): "Agentes sociales locales. La necesidad de un modelo integrado para el desarrollo socioeconómico del territorio", Arxius de Ciències Socials, $\mathrm{n}^{\mathrm{o}}$ 21, p. 7-20.

COMÍN, COMÍN, F. (2010): "Las fases Históricas de la Seguridad Social en España (1900-2000)", en De la beneficencia al Estado de Bienestar, pasando por los seguros sociales, Revista de la Historia Economía y de lo Empresa, n IV, p. 65-90.

LÓPEZ, J., (1973): Historia urgente de Cajas de Ahorro y Montes de Piedad en España, Madrid, CECA, 322 p.

SANCHIS, R., ORTIZ, V., SANZ, R., J. SANCHIS, J., BENEITO, V., (2008): Sentirte. Caixa Ontinyent 1884-2009, Ontinyent, Caixa Ontinyent, 235 p.

VILAR RODRÍGUEZ, M. (2010): "La cobertura social al margen del Estado: Asociacionismo obrero y socorros mutuos en Galicia (1839-1935)", en De la beneficencia al Estado de Bienestar, pasando por los seguros sociales, Revista de la Historia Economía y de lo Empresa, n IV, p. 179-206. 